(c) 2004 International Press

Adv. Theor. Math. Phys. 7 (2004) 807-829

\title{
A New Approach to Quantising Systems: II. Quantising on a Category of Sets
}

\author{
C.J. Isham \\ The Blackett Laboratory \\ Imperial College of Science, Technology \& Medicine \\ South Kensington \\ London SW7 2BZ \\ c.isham@imperial.ac.uk
}

\begin{abstract}
In [1], a new approach was suggested for finding quantum structures that might, in particular, be used in potential approaches to quantum gravity that involve non-manifold models for space and/or space-time (for example, causal sets). This involved developing a procedure for quantising a system whose configuration space - or historytheory analogue - is the set of objects in a (small) category $\mathcal{Q}$. In the present paper, we show how this theory can be applied to the special case when $\mathcal{Q}$ is a category of sets.
\end{abstract}

e-print archive: http://lanl.arXiv.org/abs/gr-qc/0304077 


\section{Introduction}

In [1], hereafter referred to as I., a new approach was developed for constructing quantum theories that might, in particular, be used in potential approaches to quantum gravity that involve non-manifold models for space and/or spacetime. This could include things like 'quantum topology', 'quantum manifolds', 'quantum causal sets' etc.

The starting point is the observation that, in each such example, the entities of interest are the objects of a category whose arrows are structurepreserving maps. This suggested tackling the more general problem of finding a quantum theory of a system whose configuration space (or historytheory analogue) is the set of objects $\operatorname{Ob}(\mathcal{Q})$ of an arbitrary small category $\mathcal{Q}$. A general solution to this problem of 'quantising on a category' was presented in I., and in the present paper we show how this theory works in practice for categories of sets.

First, a summary of the results obtained in $\mathbf{I}$. The methods used were motivated by considering, as an analogy, a system whose configuration spaces is a homogeneous space $Q \simeq G / H$, where $G$ and $H$ are Lie groups. In particular, the analogue of the group $G$ is chosen to be the $\operatorname{set} \operatorname{AF}(\mathcal{Q})$ of 'arrow fields' on $\mathcal{Q}$, where an arrow field is defined to be a map $X: \mathrm{Ob}(\mathcal{Q}) \rightarrow$ $\operatorname{Hom}(\mathcal{Q})$ (the arrows of $\mathcal{Q}$ ) such that, for each $A \in \mathrm{Ob}(\mathcal{Q})$, the domain of $X(A)$ is $A$; thus $X(A): A \rightarrow B$ for some $B \in \mathrm{Ob}(\mathcal{Q})$. A simple, and useful, example of an arrow field is when all but one of the arrows $X(A)$, $A \in \mathrm{Ob}(\mathcal{Q})$, is the identity $\operatorname{id}_{A}: A \rightarrow A$. Specifically, for each arrow $f$, an arrow field $X_{f}$ can be defined by

$$
X_{f}(A):= \begin{cases}f & \text { if Dom } f=A \\ \operatorname{id}_{A} & \text { otherwise }\end{cases}
$$

for all $A \in \mathrm{Ob}(\mathcal{Q})$.

A crucial property of arrow fields is that they form a monoid ${ }^{1}$. Specifically: if $X_{1}, X_{2} \in \operatorname{AF}(\mathcal{Q})$, we construct an arrow field $X_{2} \& X_{1}$ by defining the arrow $\left(X_{2} \& X_{1}\right)(A), A \in \mathrm{Ob}(\mathcal{Q})$, to be the composition of the arrow $X_{1}(A)$ with the arrow obtained by evaluating $X_{2}$ on the range of $X_{1}(A)$ :

$$
\left(X_{2} \& X_{1}\right)(A):=X_{2}\left(\operatorname{Ran} X_{1}(A)\right) \circ X_{1}(A) .
$$

Put more simply, if $X_{1}(A): A \rightarrow B$, then $\left(X_{2} \& X_{1}\right)(A):=X_{2}(B) \circ X_{1}(A)$.

\footnotetext{
${ }^{1} \mathrm{~A}$ monoid is a semigroup with a unit element. In the present case, the unit element is the arrow field $\iota$ defined by $\iota(A):=\operatorname{id}_{A}$ for all $A \in \mathrm{Ob}(\mathcal{Q})$.
} 
An action of the monoid $\operatorname{AF}(\mathcal{Q})$ on $\mathrm{Ob}(\mathcal{Q})$ can be defined by $\ell_{X}(A):=$ $\operatorname{Ran} X(A)$, so that if $X(A): A \rightarrow B$ then $\ell_{X}(A)=B$. This action was used in I. to construct an operator (anti-) representation of $\operatorname{AF}(\mathcal{Q})$ on complexvalued functions on $\mathrm{Ob}(\mathcal{Q})$ by defining, for all $A \in \mathrm{Ob}(\mathcal{Q})$,

$$
(\hat{a}(X) \psi)(A):=\psi\left(\ell_{X} A\right) .
$$

These operators satisfy

$$
\hat{a}\left(X_{2}\right) \hat{a}\left(X_{1}\right)=\hat{a}\left(X_{1} \& X_{2}\right)
$$

for all arrow fields $X_{1}, X_{2}$. If Eq. (3) is applied to the special arrow fields $X_{f}$ in Eq. (1) then, defining $\hat{a}(f):=\hat{a}\left(X_{f}\right)$, we get

$$
(\hat{a}(f) \psi)(A)= \begin{cases}\psi(\operatorname{Ran} f) & \text { if Dom } f=A \\ \psi(A) & \text { otherwise. }\end{cases}
$$

The inner product on the quantum state functions is

$$
\langle\phi \mid \psi\rangle:=\int_{\mathrm{Ob}(\mathcal{Q})} d \mu(A) \phi^{*}(A) \psi(A)
$$

for some measure $\mu$ on $\operatorname{Ob}(\mathcal{Q})$. If $\operatorname{Ob}(\mathcal{Q})$ is finite, or countably infinite, a natural choice for the inner product is

$$
\langle\phi \mid \psi\rangle:=\sum_{A \in \mathrm{Ob}(\mathcal{Q})} \phi(A)^{*} \psi(A) .
$$

The 'configuration' space variables are members of the space $F(\mathrm{Ob}(\mathcal{Q}), \mathbb{R})$ of all real-valued functions on $\mathrm{Ob}(\mathcal{Q})$, and unitary operators $\hat{V}(\beta), \beta \in$ $F(\mathrm{Ob}(\mathcal{Q}), \mathbb{R})$, are defined by

$$
(\hat{V}(\beta) \psi)(A):=e^{-i \beta(A)} \psi(A)
$$

for all $A \in \mathrm{Ob}(\mathcal{Q})$.

The operators $\hat{a}(X), X \in \operatorname{AF}(\mathcal{Q})$, and $\hat{V}(\beta), \beta \in F(\operatorname{Ob}(\mathcal{Q}), \mathbb{R})$, satisfy the relations

$$
\begin{aligned}
\hat{a}\left(X_{2}\right) \hat{a}\left(X_{1}\right) & =\hat{a}\left(X_{1} \& X_{2}\right) \\
\hat{V}\left(\beta_{1}\right) \hat{V}\left(\beta_{2}\right) & =\hat{V}\left(\beta_{1}+\beta_{2}\right) \\
\hat{a}(X) \hat{V}(\beta) & =\hat{V}\left(\beta \circ \ell_{X}\right) \hat{a}(X)
\end{aligned}
$$

and, as such, constitute a representation of the 'category quantisation monoid', which is defined to be the semi-direct product $\operatorname{AF}(\mathcal{Q}) \times_{\ell} F(\mathrm{Ob}(\mathcal{Q}), \mathbb{R})$. In 
general, the possible quantisations of the system are deemed to be given by the irreducible, faithful representations of this monoid.

The representation in Eq. (3) and Eq. (8) is inadequate because it fails to separate arrows ${ }^{2}$ with the same domain and range. This can be rectified by introducing a suitable bundle of Hilbert spaces, $A \rightsquigarrow \mathcal{K}[A]$, with linear 'multipliers' $m(X, A): \mathcal{K}\left[\ell_{X} A\right] \rightarrow \mathcal{K}[A]$. The arrow-field operators $\hat{a}(X)$, $X \in \operatorname{AF}(\mathcal{Q})$, are now defined as

$$
(\hat{a}(X) \psi)(A):=m(X, A) \psi\left(\ell_{X} A\right)
$$

and satisfy Eq. (9) provided the multipliers are subject to the conditions

$$
m(Y \& X, A)=m(X, A) m\left(Y, \ell_{X} A\right)
$$

for all $X, Y \in \operatorname{AF}(\mathcal{Q})$ and $A \in \operatorname{Ob}(\mathcal{Q})$. Note that if the arrow fields $X, Y$ are such that, for some $A, X(A): A \rightarrow A$ and $Y(A): A \rightarrow A$, then Eq. (13) gives

$$
m(Y \& X, A)=m(X, A) m(Y, A)
$$

which corresponds to an anti-representation of the monoid $\operatorname{Hom}(A, A)$ on the Hilbert space $\mathcal{K}[A]$.

As explained in I., a natural way of generating structures of this type is to start with a presheaf of Hilbert spaces over $\mathrm{Ob}(\mathcal{Q})$, so that any arrow $f: A \rightarrow B$ is 'covered' by a linear map $\kappa(f): \mathcal{K}[B] \rightarrow \mathcal{K}[A]$, and then to define a multiplier by $m(X, A):=\kappa(X(A))$ for all $X \in \operatorname{AF}(\mathcal{Q}), A \in \mathrm{Ob}(\mathcal{Q})$. The quantum states are defined to be cross-sections of the Hilbert bundle, with the inner product

$$
\langle\phi \mid \psi\rangle:=\int_{\mathrm{Ob}(\mathcal{Q})} d \mu(A)\langle\phi(A), \psi(A)\rangle_{\mathcal{K}[A]}
$$

where $\langle\cdot, \cdot\rangle_{\mathcal{K}[A]}$ denotes the inner product in the Hilbert space $\mathcal{K}[A]$, and $\mu$ is a measure on $\operatorname{Ob}(\mathcal{Q})$. The operators $\hat{V}(\beta)$ are defined as in Eq. (8).

The aim of the present paper is to give some examples of this scheme to illustrate the basic ideas. We start in Section 2 with the simple case when $\mathcal{Q}$ is a partially-ordered set whose objects are points with no internal structure, and multipliers are not needed. The heart of the paper is Section 3 which contains a discussion of the quantisation on a category of sets. Section 3.1 focusses on a category of finite sets, and we show how to construct explicit multiplier representations that separate the arrows in the category.

\footnotetext{
${ }^{2}$ Two arrows $f, g$ with the same domain and range are said to beseparated in the quantum theory if $\hat{a}\left(X_{f}\right) \neq \hat{a}\left(X_{g}\right)$.
} 
In Section 3.2 we discuss briefly how these methods can be extended to nonfinite sets with the aid of categories of measure spaces. Then, after a few cautionary remarks about adapting the scheme to categories of causal sets, we give a complete quantisation in Section 3.4 of a very simple category with just two causal sets. In Section 4 we show how certain special operations on causal sets - for example, taking the linear sum of two of them-can be incorporated naturally into the scheme, as can the lattice operations on a lattice. Finally, in Section 5 we return to a poset category to show how standard quantum field theory is another special example of the scheme.

\section{Some Simple Examples}

\subsection{The Simplest Example}

The simplest example is when $\mathcal{Q}$ is a monoid $M$ considered as a category. There is just one object \#, and the arrows are in one-to-one correspondence with the elements of $M$, with arrow composition being monoid multiplication. An arrow field is simply an arrow; i.e., an element of $M$. A quantum theory on this category can be found by associating with \# the Hilbert space $\mathcal{K}$ of any faithful, irreducible representation $m \mapsto \hat{R}(m)$ of $M$. The state vectors are functions from $\#$ to $\mathcal{K}$, and are hence just elements of $\mathcal{K}$. If $m$ is an arrow-field/arrow/monoid element, then $\hat{a}(m):=\hat{R}(m)$. The faithfulness of the representation guarantees that it separates the arrows, i.e., the elements of $M$.

\section{$2.2 \quad$ A Poset Category}

A monoid considered as a category has only one object, but many arrows. At the other extreme is a partially ordered set (poset) $P$ considered as a category. The objects are the elements of $P$, and there is an arrow $o_{p, q}$ : $p \rightarrow q$ if, and only if, $p \leq q$. Thus there is at most one arrow between each pair of objects - in particular, $\operatorname{Hom}(p, p)=\left\{\operatorname{id}_{p}\right\}$ for all $p \in P$-but many objects.

It is important to be clear about the physical situations that could be represented by such a model. For example, consider a causal set theory of the type discussed recently by Dowker ${ }^{3}$ in which, in the language of the present paper, the objects of the category $\mathcal{Q}$ are subsets of a single, 'master' causal

\footnotetext{
${ }^{3}$ Fay Dowker, private communication.
} 
set $\Gamma$. Superficially, this category might look like a poset, with the ordering being subset inclusion. However, this is misleading since each object $A \subset \Gamma$ has an internal structure, as reflected in the monoid $\operatorname{Hom}(A, A)$ of orderpreserving maps from $A$ to itself, and one would want to represent this in the quantum theory. More generally, if $A$ and $B$ are any non-trivial causal subsets of $\Gamma$ with $A \subset B$, then $\operatorname{Hom}(A, B)$ typically contains more than just the subset inclusion map $i_{A, B}: A \subset B$. For example, if $\alpha \in \operatorname{Hom}(A, A)$ and $\beta \in \operatorname{Hom}(B, B)$, then

$$
\beta \circ i_{A, B} \circ \alpha: A \rightarrow B
$$

belongs to $\operatorname{Hom}(A, B)$. Thus $\mathcal{Q}$ is not just a poset category.

When we do have a genuine poset category $P$, there is at most one arrow between any pair of objects, which means that to separate arrows in the quantum theory it suffices to let the state space be just complex-valued functions on $P$. Then, if $p \leq q$, the operator $\hat{a}(p, q):=\hat{a}\left(X_{o_{p, q}}\right)$ associated with the arrow $o_{p, q}: p \rightarrow q$ is (see Eq. (5))

$$
(\hat{a}(p, q) \psi)(r)= \begin{cases}\psi(q) & \text { if } p=r ; \\ \psi(r) & \text { otherwise. }\end{cases}
$$

As in Eq. (8), functions $\beta: P \rightarrow \mathbb{R}$ are represented by

$$
(\hat{V}(\beta) \psi)(r):=e^{-i \beta(r)} \psi(r)
$$

for all $r \in P$. The natural inner product is the one in Eq. (7) provided $P$ has at most a countable number of elements. For a system whose configuration space (or history-theory analogue) is a poset with a finite number, $n$, of elements, the quantum state space is just $\mathbb{C}^{n}$.

\subsection{Quantum Theory on $\mathbb{N}$}

A simple example of a poset is a finite chain with the Hasse diagram

$$
0 \rightarrow 1 \rightarrow 2 \rightarrow \cdots \rightarrow n-1
$$

in which the only arrows between objects/points are those shown in Eq. (19), plus combinations of them, plus the identity arrow at each object. The quantisation goes ahead as above, and the Hilbert state space is $\mathbb{C}^{n}$.

More interesting is the generalisation with a countably infinite number of objects

$$
0 \rightarrow 1 \rightarrow 2 \rightarrow \cdots \rightarrow n-1 \rightarrow \cdots
$$


so that $\operatorname{Ob}(\mathcal{Q})$ is the natural numbers $\mathbb{N}$. In this case, there is a special family of arrow fields $X^{m}, m \in \mathbb{N}$, defined on all $n \in \mathbb{N}$ by

$$
X^{m}(n):=n \rightarrow(n+m)
$$

where the right hand side denotes the combination of $m$ successive arrows in the Hasse diagram of Eq. (20). Clearly

$$
X^{m_{1}} \& X^{m_{2}}=X^{m_{1}+m_{2}},
$$

and hence the set of all arrow fields of this type is a submonoid of $\operatorname{AF}(\mathcal{Q})$. It is isomorphic to the additive monoid $\mathbb{N}$.

This submonoid acts on state functions as

$$
\left(\hat{a}\left(X^{m}\right) \psi\right)(n)=\psi(n+m),
$$

for all $n \in \mathbb{N}$. Together with the representation Eq. (8) of the space of functions $F(\mathbb{N}, \mathbb{R})$, this gives an irreducible representation of the semi-direct product monoid $\mathbb{N} \times_{\ell} F(\mathbb{N}, \mathbb{R})$. This is a submonoid of the full category quantisation monoid $\operatorname{AF}(\mathcal{Q}) \times_{\ell} F(\mathbb{N}, \mathbb{R})$. This is an example of the general fact that for an arbitrary small category $\mathcal{Q}$ there may well be submonoids of $\operatorname{AF}(\mathcal{Q}) \times_{\ell} F(\mathrm{Ob}(\mathcal{Q}), \mathbb{R})$ for which an irreducible representation of $\operatorname{AF}(\mathcal{Q}) \times_{\ell}$ $F(\mathrm{Ob}(\mathcal{Q}), \mathbb{R})$ remains irreducible. This is analogous to what happens when $Q \simeq G / H$, where an irreducible representation of the semi-direct product $\operatorname{Diff}(Q) \times{ }_{d} C^{\infty}(Q, \mathbb{R})$ remains irreducible when restricted to the subgroup $G \times{ }_{\tau} W$, where $W$ is a special finite-dimensional subspace of $C^{\infty}(Q, \mathbb{R}$ ) (for more details see $\mathbf{I}$ ).

Note that the example in Eq. (23) illustrates something that is missing for a general small category: namely, the existence of arrow fields that give the 'same' arrow at each object.

We could write down what is superficially the same category as in Eq. (20) but with the object $n$ now being identified with the set $\{0,1,2, \ldots, n-$ 1\}. Thus the Hasse diagram of Eq. (20) is replaced with

$$
\{0\} \rightarrow\{0,1\} \rightarrow\{0,1,2\} \rightarrow \ldots
$$

where the arrow between each pair of objects is now interpreted as a subset embedding. This gives rise to a quantum theory of finite sets in which the number of elements in a set can change.

However, the situation here is essentially the same as that mentioned in Section 2.2 in the context of Fay Dowker's model. Specifically, if each object $A$ is viewed as an explicit set $\{0,1, \ldots, \ldots, n-1\}$, then it now has an internal 
structure, as reflected in the non-triviality of $\operatorname{Hom}(A, A)$. Thus the category is different from the starting example in Eq. (20), and the quantisation should reflect this. In particular, the monoids $\operatorname{Hom}(A, A)$ should be represented faithfully, and hence separate arrows of the type $\beta \circ i_{A, B} \circ \alpha: A \rightarrow B$, where $\beta: B \rightarrow B, \alpha: A \rightarrow A$, and $i_{A, B}: A \rightarrow B$ is the concatenation of the appropriate number of embeddings in Eq. (24). This requires the introduction of non-trivial Hilbert spaces $\mathcal{K}[A]$ and associated multipliers.

\section{Quantising on a Category of Sets}

\subsection{The Example Of Finite Sets}

\subsubsection{Constructing the Multipliers}

The physical examples in which we are most interested arise when $\mathcal{Q}$ is a small category of causal sets (i.e., posets), or topological spaces, or differentiable manifolds, or any other such mathematical entities that are potential models for space-time, or space. The objects in these categories are sets with structure, and the arrows are structure-preserving maps. Here we shall discuss in detail the most basic situation of this type, which is when $\mathcal{Q}$ is a small category of sets, and the arrows are any maps between a pair of sets. The resulting theory can then be used as a stepping stone for handling categories whose objects have a more specific structure, and whose morphisms are therefore more restricted.

The operators $\hat{a}(X)$ that represent arrow fields are defined in Eq. (12), and - as in $\mathbf{I}$. - we shall assume that the multiplier $m(X, A): \mathcal{K}\left[\ell_{X} A\right] \rightarrow$ $\mathcal{K}[A]$ is obtained from a family of linear maps $\kappa(f): \mathcal{K}[\operatorname{Ran} f] \rightarrow \mathcal{K}[\operatorname{Dom} f]$, $f \in \operatorname{Hom}(\mathcal{Q})$, such that $m(X, A)=\kappa(X(A))$ for all $X \in \operatorname{AF}(\mathcal{Q})$ and $A \in$ $\mathrm{Ob}(\mathcal{Q})$. In order to ensure the multiplier conditions Eq. (14), these maps must satisfy the coherence conditions

$$
\kappa(g \circ f)=\kappa(f) \kappa(g): \mathcal{K}[C] \rightarrow \mathcal{K}[A]
$$

for any $f: A \rightarrow B$ and $g: B \rightarrow C$. As discussed in I., the conditions Eq. (25) are equivalent to the existence of a presheaf of Hilbert spaces on $\mathcal{Q}$, where a function $f: A \rightarrow B$ is 'covered' by the linear map $\kappa(f): \mathcal{K}[B] \rightarrow \mathcal{K}[A]$.

A necessary condition for the quantum theory to separate arrows is that each Hilbert space $\mathcal{K}[A], A \in \mathrm{Ob}(\mathcal{Q})$, must carry a faithful representation of the monoid $\operatorname{Map}(A, A)(=\operatorname{Hom}(A, A))$ of all maps from the set $A$ to itself. It would be very desirable if we could find a suitable presheaf that is generic 
in the sense that it can be defined for any small category of sets without invoking the specific details of the category.

One vector space that is naturally associated with any set $A$, is the space $F(A, \mathbb{C})$ of complex-valued functions on $A$. Then, to each arrow/function $f$ : $A \rightarrow B$, there corresponds an obvious linear map $\kappa(f): F(B, \mathbb{C}) \rightarrow F(A, \mathbb{C})$ defined on $v \in F(B, \mathbb{C})$ by

$$
(\kappa(f) v)(a):=v(f(a))
$$

for all $a \in A$.

To check the coherence conditions Eq. (25), suppose $f: A \rightarrow B$ and $g: B \rightarrow C$, and let $v \in F(C, \mathbb{C})$. Then

$$
(\kappa(f) \kappa(g) v)[a]=(\kappa(g) v)[f(a)]=v[g(f(a)]
$$

However, $g(f(a))=g \circ f(a)$, and so Eq. (27) gives

$$
(\kappa(f) \kappa(g) v)[a]=v[g \circ f(a)]=(\kappa(g \circ f) v)[a]
$$

and hence $\kappa(f) \kappa(g)=\kappa(g \circ f)$, as required.

As it stands, $F(A, \mathbb{C})$ is merely a complex vector space. However, if $A$ is a finite set, there is a vector space isomorphism $F(A, \mathbb{C}) \simeq \mathbb{C}^{|A|}$, where $|A|$ denotes the number of elements in $A$. The standard inner product on

$\mathbb{C}^{|A|}$ can then be used to equip $F(A, \mathbb{C})$ with an inner product. In [2], this way of quantising on a category of finite sets is rederived using a different technique based on taking state vectors as complex-valued functions on the set of arrows $\operatorname{Hom}(\mathcal{Q})$.

If $A$ is a countable set, a natural choice for $\mathcal{K}[A]$ is the space $\ell^{2}(A)$ of square-summable sequences of complex numbers indexed by the elements of $A$. However, care is now needed since the map in Eq. (26) may not take a square-summable sequence into one that is also square summable; for example, this happens if $f: A \rightarrow B$ is a constant function. In this Section, we shall restrict our attention to categories of finite sets, so everything is well defined.

\subsubsection{The Detailed Calculations}

If $A$ is a finite set, then any $\vec{v} \in \mathbb{C}^{|A|}$, can be expressed as a sum

$$
\vec{v}=\sum_{a \in A} v_{a} \vec{a}
$$


where $\vec{a}$ denotes the element $a \in A$ thought of as a basis vector for $\mathbb{C}^{|A|}$. The expansion coefficients $v_{a} \in \mathbb{C}, a \in A$, are given in terms of the inner product $\langle\cdot, \cdot\rangle_{\mathbb{C}^{|A|}}$ as

$$
v_{a}=\langle\vec{a}, \vec{v}\rangle_{\mathbb{C}^{|A|}} .
$$

In this notation, the linear transformation $\kappa(f): \mathbb{C}^{|B|} \rightarrow \mathbb{C}^{|A|}$ in Eq. (26), associated with $f: A \rightarrow B$, is

$$
\kappa(f): \sum_{b \in B} v_{b} \vec{b} \mapsto \sum_{a \in A} v_{f(a)} \vec{a} .
$$

For each $b \in \operatorname{Ran} f \subset B$, and for all $a \in f^{-1}\{b\}$, the value of $v_{f(a)}$ on the right hand side of Eq. (31) is constant, and equal to $v_{b}$. Thus, as $a$ ranges of $f^{-1}\{b\}$ we get a linear sum of the corresponding basis vectors $\vec{a}$. This can be turned into a sum over $b \in B$ by noting that only those $b$ arise that are in the image of $f$. Thus the map in Eq. (31) can be rewritten as

$$
\kappa(f): \sum_{b \in B} v_{b} \vec{b} \mapsto \sum_{b \in B} v_{b} \overline{f^{-1}\{b\}}
$$

where

$$
\overline{f^{-1}\{b\}}:=\sum_{a \in f^{-1}\{b\}} \vec{a}
$$

denotes the sum in $\mathbb{C}^{|A|}$ of the basis vectors associated with those elements in $A$ that are mapped by $f$ to $b \in B$. From this perspective, the fundamental coherence condition Eq. (25) follows from the basic property of inverse set maps:

$$
f^{-1}\left(g^{-1}\{c\}\right)=(g \circ f)^{-1}\{c\}
$$

for all $f: A \rightarrow B$ and $g: B \rightarrow C$.

Since $\kappa(f): \mathbb{C}^{|B|} \rightarrow \mathbb{C}^{|A|}$ is linear, it can be written as

$$
\kappa(f): \sum_{b \in B} v_{b} \vec{b} \mapsto \sum_{b \in B} v_{b} \kappa(f) \vec{b},
$$

and so another way of specifying $\kappa(f)$ in Eq. (32) is in terms of its action on the basis vectors $\{\vec{b} \mid b \in B\}$ of $\mathbb{C}^{|B|}$ :

$$
\kappa(f) \vec{b}:=\overline{f^{-1}\{b\}}=\sum_{a \in f^{-1}\{b\}} \vec{a} .
$$

In particular, the matrix elements of $\kappa(f): \mathbb{C}^{|B|} \rightarrow \mathbb{C}^{|A|}$ are

$$
\langle\vec{a}, \kappa(f) \vec{b}\rangle_{\mathbb{C}^{|A|}}= \begin{cases}1 & \text { if } a \in f^{-1}\{b\}, \text { i.e., if } b=f(a) ; \\ 0 & \text { otherwise. }\end{cases}
$$

This shows that, as desired, the multiplier $\kappa$ separates arrows that have the same domain and range. 


\subsubsection{The Adjoint of $k$}

The linear map $\kappa(f): \mathcal{K}[\operatorname{Ran} f] \rightarrow \mathcal{K}[\operatorname{Dom} f]$ is used in the definition Eq. (12) of the arrow field operator $\hat{a}(X)$, with $m(X, A):=\kappa(X(A))$. On the other hand, as shown in I., the corresponding expression (for finite sets) for the adjoint $\hat{a}(X)^{\dagger}$ is

$$
\left(\hat{a}(X)^{\dagger} \psi\right)(B)=\sum_{A \in \ell_{X}^{-1}\{B\}} \kappa(X(A))^{\dagger} \psi(A)
$$

where $\kappa(X(A))^{\dagger}: \mathcal{K}[A] \rightarrow \mathcal{K}\left[\ell_{X} A\right]$ is the adjoint of the linear map $\kappa(X(A)):$ $\mathcal{K}\left[\ell_{X} A\right] \rightarrow \mathcal{K}[A]$.

To compute $\kappa(X(A))^{\dagger}$ explicitly, note that if $f: A \rightarrow B$, then $\kappa(f)^{\dagger}$ : $\mathbb{C}^{|A|} \rightarrow \mathbb{C}^{|B|}$ is defined by

$$
\left\langle\kappa(f)^{\dagger} \vec{w}, \vec{v}\right\rangle_{\mathbb{C}^{|B|}}:=\langle\vec{w}, \kappa(f) \vec{v}\rangle_{\mathbb{C}^{|A|}}
$$

for all $\vec{v} \in \mathbb{C}^{|B|}$ and $\vec{w} \in \mathbb{C}^{|A|}$. With respect to the basis $\{\vec{b} \mid b \in B\}$ of $\mathbb{C}^{|B|}$, we have

$$
\begin{aligned}
\left(\kappa(f)^{\dagger} \vec{w}\right)_{b} & =\left\langle\kappa(f)^{\dagger} \vec{w}, \vec{b}\right\rangle_{\mathbb{C}^{|B|}}=\langle\vec{w}, \kappa(f) \vec{b}\rangle_{\mathbb{C}^{|A|}}=\left\langle\vec{w}, \sum_{a \in f^{-1}\{b\}} \vec{a}\right\rangle_{\mathbb{C}^{|A|}} \\
& =\sum_{a \in f^{-1}\{b\}} w_{a}
\end{aligned}
$$

which gives the expression for $\kappa(f)^{\dagger}: \mathbb{C}^{|A|} \rightarrow \mathbb{C}^{|B|}$ :

$$
\begin{aligned}
\kappa(f)^{\dagger} \vec{w} & =\sum_{b \in B} \sum_{a \in f^{-1}\{b\}} w_{a} \vec{b}=\sum_{b \in B} \sum_{a \in A} w_{a} \delta_{b, f(a)} \vec{b} \\
& =\sum_{a \in A} w_{a} \overline{f(a)} .
\end{aligned}
$$

for all $\vec{w} \in \mathbb{C}^{|A|}$.

\subsection{Quantum Measure Theory}

\subsubsection{The General Idea}

A more general way of associating Hilbert spaces $\mathcal{K}[A]$ with the sets $A \in$ $\mathrm{Ob}(\mathcal{Q})$ is if $\mathcal{Q}$ is a category of measure spaces in which each set $A \in \mathrm{Ob}(\mathcal{Q})$ 
is equipped with a measure $\mu_{A}$ on a $\sigma$-field of subsets of $A{ }^{4}$ The arrows in this category are functions $f: A \rightarrow B$ that are measurable with respect to the measure structures on $A$ and $B .^{5}$ We can then form the bundle of Hilbert spaces $A \rightsquigarrow L^{2}\left(A, d \mu_{A}\right)$ and try using this as the quantum bundle $A \rightsquigarrow \mathcal{K}[A]$. For example, if $A$ is a finite set, a natural choice for $\mu_{A}$ is $\mu_{A}(M)=|M|$, for any subset $M$ of $A$. The Hilbert space $L^{2}\left(A, d \mu_{A}\right)$ is then isomorphic to $\mathbb{C}^{|A|}$, and we recover the situation discussed in Section 3.1.

We now proceed as in Section 3.1.1 and use Eq. (26) to define the linear map $\kappa(f): L^{2}\left(B, d \mu_{B}\right) \rightarrow L^{2}\left(A, d \mu_{A}\right)$ for each arrow (measurable map) $f: A \rightarrow B$. However, care is needed if some of the Hilbert spaces $L^{2}\left(A, d \mu_{A}\right)$, $A \in \mathrm{Ob}(\mathcal{Q})$, are infinite dimensional since the map $\kappa(f)$ could then be unbounded. This is similar to the problem mentioned earlier in the context of using Eq. (26) when some of the sets $A$ are countably infinite.

This situation can be tackled in various ways. One is to allow the maps $\kappa(f)$ to be unbounded, with domains that are dense subsets of the appropriate Hilbert spaces. A more attractive scheme is to restrict the arrows in the category to be measurable maps for which these problems do not arise. Of course, if such a quantum scheme is to be applied to a concrete physical problem, there need to be good physical reasons for making restrictions of this type.

\subsubsection{The Special Case of a Category of Groups}

One situation where measures arise naturally is if $\mathcal{Q}$ is a small category of locally compact topological groups, with the arrows being continuous group homomorphisms. Each such group $G$ has a unique Haar measure $\mu_{G}$, and this gives a preferred choice for the Hilbert spaces $\mathcal{K}[G]$ : namely, $L^{2}\left(G, d \mu_{G}\right)$.

Then, if $\phi: G \rightarrow H$ is a continuous homomorphism, we follow the example of Eq. (26) and define a linear map $\kappa(\phi): L^{2}\left(H, d \mu_{H}\right) \rightarrow L^{2}\left(G, d \mu_{G}\right)$ by

$$
(\kappa(\phi) v)(g):=v(\phi(g))
$$

for all $g \in G$ and $v \in L^{2}\left(H, d \mu_{H}\right)$ (once again, care is needed if any of the Hilbert spaces have infinite dimension).

\footnotetext{
${ }^{4}$ Note that a scheme of this type allows for the possibility that the objects in the category may include more than one copy of the same set equipped with different measures (and associated fields of measurable sets) on that set.

${ }^{5}$ It may be appropriate to identify functions $f_{1}, f_{2}: A \rightarrow B$ that are equal up to a set of $\mu_{A}$-measure zero.
} 
An important property of the Hilbert space $L^{2}\left(G, d \mu_{G}\right)$ is that it carries the regular representation of $G$ defined by

$$
\left(l_{g} v\right)\left(g_{0}\right):=v\left(g_{0} g\right)
$$

Although it is not strictly relevant to our needs, we note that the maps $\kappa(\phi): L^{2}\left(H, d \mu_{H}\right) \rightarrow L^{2}\left(G, d \mu_{G}\right)$ are actually intertwining operators for the regular representations on the two groups. Thus, if $\psi \in L^{2}\left(H, d \mu_{H}\right)$ and $\phi: G \rightarrow H$ is any continuous group homomorphism, we have

$$
\begin{aligned}
\left(l_{g} \kappa(\phi) \psi\right)\left(g_{0}\right) & =(\kappa(\phi) \psi)\left(g_{0} g\right)=\psi\left[\phi\left(g_{0} g\right)\right] \\
& =\psi\left[\phi\left(g_{0}\right) \phi(g)\right]=\left(l_{\phi(g)} \psi\right)\left[\phi\left(g_{0}\right)\right] \\
& =\left(\kappa(\phi) \ell_{\phi(g)} \psi\right)\left(g_{0}\right)
\end{aligned}
$$

for all $g_{0}, g \in G$. This gives the intertwining relations

$$
l_{g} \kappa(\phi)=\kappa(\phi) l_{\phi(g)}
$$

corresponding to the commutative diagram

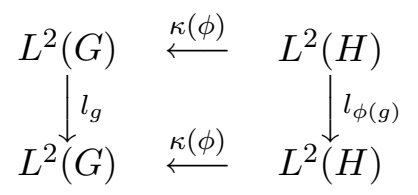

\subsection{Categories of Causal Sets or Topological Spaces}

\subsubsection{Cautionary Remarks}

Let us turn now to the problem of quantising on a category $\mathcal{Q}$ of causal sets, or topological spaces, or manifolds, etc., in which the arrows $f: A \rightarrow B$ are structure-preserving functions. As a first try we could forget about this extra structure and use the category $\mathcal{Q}_{S}$ whose objects are defined to be the same as those of $\mathcal{Q}$ but whose arrows $f: A \rightarrow B$ are now all functions from $A$ to $B$, not just those that are structure preserving.

Suppose that we succeed in constructing a proper quantisation on $\mathcal{Q}_{S}$ by finding a representation of the category quantisation monoid that is both irreducible and faithful. Then since each arrow in $\mathcal{Q}$ is also a function between sets, and hence an arrow in $\mathcal{Q}_{S}$, it follows that each arrow field in $\operatorname{AF}(\mathcal{Q})$ will also belong to $\operatorname{AF}\left(\mathcal{Q}_{S}\right)$, and will hence be represented in the quantum theory on $\mathcal{Q}_{S}$. Furthermore, since the quantum theory on $\mathcal{Q}_{S}$ separates arrows, a fortiori the same will be true for the subset of arrows that belong to 
$\mathcal{Q}$. Therefore, the representation of the category quantisation monoid of $\mathcal{Q}$ obtained in this way will certainly be faithful.

However, there is no guarantee that this representation is irreducible. For example, if $A$ is a finite set, the Hilbert space $\mathbb{C}^{|A|}$ carries an irreducible representation of the monoid $\operatorname{Map}(A, A)$, but it may be reducible with respect to the submonoid of order-preserving maps, or continuous maps, etc. If this happens for at least one object $A \in \mathrm{Ob}(\mathcal{Q})$, then the representation of the category quantisation monoid of $\mathcal{Q}$ will no longer be irreducible ${ }^{6}$. And even if the representation of $\operatorname{Hom}(A, A)$ is irreducible for all $A \in \mathrm{Ob}(\mathcal{Q})$, the representation of the category quantisation monoid could still be reducible if there are not enough arrows between pairs of different objects.

It might be possible to say something about this issue in a general way, but probably it will need to be tackled on a case-by-case basis, with each specific category $\mathcal{Q}$ of physical interest being examined in detail.

\subsection{A Very Simple Example}

\subsubsection{Quantisation with State Functions}

We will now illustrate the general scheme with the aid of a very simple example of a category with just two objects: the causal sets (posets) $A:=$ $\{a\}$, and $B:=\{b, c\}$ with $b \leq c$. The arrows are order-preserving maps, and there are two such $f_{1}, f_{2}$ from $A$ to $B$ defined by

$$
\begin{aligned}
& f_{1}(a):=b \\
& f_{2}(a):=c
\end{aligned}
$$

respectively, and one arrow $g$ from $B$ to $A$ defined by

$$
g(b):=a, g(c):=a .
$$

A non-trivial arrow $r: B \rightarrow B$ is

$$
r(b):=b, r(c):=b
$$

which is not invertible. The same applies to the other non-trivial arrow $s: B \rightarrow B$ defined by

$$
s(b):=c, s(c):=c .
$$

\footnotetext{
${ }^{6}$ It is being assumed here that $\mathcal{Q}$ has a finite or countable number of objects with an associated inner product Eq. (7). The discussion needs to be extended in an obvious way for the inner product in Eq. (15) with a general measure $\mu$ on $\mathrm{Ob}(\mathcal{Q})$.
} 
If we forget the causal/ordering structure on the set $B$, then an additional arrow $p: B \rightarrow B$ can be defined by

$$
p(b):=c, p(c):=b
$$

which is the non-trivial element of the permutation group $\mathbb{Z}_{2}$ of the set $B$. It is not, however, an arrow in the category $\mathcal{Q}$ since it reverses the ordering of the elements $b, c \in B$.

In summary, we have the following sets of arrows:

$$
\begin{aligned}
\operatorname{Hom}(A, B) & =\left\{f_{1}, f_{2}\right\} \\
\operatorname{Hom}(B, A) & =\{g\} \\
\operatorname{Hom}(A, A) & =\left\{\operatorname{id}_{A}\right\} \\
\operatorname{Hom}(B, B) & =\left\{\operatorname{id}_{B}, r, s\right\}
\end{aligned}
$$

to which should be added the map $p: B \rightarrow B$ in Eq. (52) if we forget the causal structure on $B$.

According to the simple quantum scheme without multipliers, the quantum Hilbert space is $\mathbb{C}^{2}$, where $\psi \in \mathbb{C}^{2}$ is represented by the pair of complex numbers $\left(\psi_{A}, \psi_{B}\right):=(\psi(A), \psi(B))$. Then, using the definition of $\hat{a}(f):=\hat{a}\left(X_{f}\right)$ in Eq. (5), we find

$$
\begin{aligned}
\hat{a}\left(f_{1}\right):\left(\psi_{A}, \psi_{B}\right) & \mapsto\left(\psi_{B}, \psi_{B}\right) \\
\hat{a}\left(f_{2}\right):\left(\psi_{A}, \psi_{B}\right) & \mapsto\left(\psi_{B}, \psi_{B}\right) \\
\hat{a}(g):\left(\psi_{A}, \psi_{B}\right) & \mapsto\left(\psi_{A}, \psi_{A}\right) \\
\hat{a}\left(\operatorname{id}_{A}\right):\left(\psi_{A}, \psi_{B}\right) & \mapsto\left(\psi_{A}, \psi_{B}\right) .
\end{aligned}
$$

This shows explicitly the failure to separate the arrows $f_{1}: A \rightarrow B$ and $f_{2}$ : $A \rightarrow B$. Similarly, the arrows in $\operatorname{Hom}(B, B)=\left\{\mathrm{id}_{B}, r, s\right\}$ are all represented by the unit operator on $\mathbb{C}^{2}$, and are hence not separated.

Thus the simple quantum scheme cannot distinguish between the given category and the one whose objects are two singleton sets $A:=\{a\}$ and $B:=\{b\}$, with just one arrow from $A$ to $B$ and one arrow from $B$ to $A$ (plus $\operatorname{id}_{A}$ and $\operatorname{id}_{B}$ as always).

\subsubsection{Quantisation With a Multiplier}

Now consider the quantisation on the same category, but this time using a multiplier. The discussion in Section 3.1.2 of quantising on a category of finite sets, suggests that the appropriate choices for the Hilbert space fibres 
are $\mathcal{K}[A]=\mathbb{C}^{1} \simeq \mathbb{C}$, and $\mathcal{K}[B]=\mathbb{C}^{2}$. Thus the quantum state space of the system is now $\mathbb{C} \oplus \mathbb{C}^{2} \simeq \mathbb{C}^{3}$; a vector $\psi$ in this space will be denoted by $\left(\psi_{A} ; \psi_{B_{1}}, \psi_{B_{2}}\right) \in \mathbb{C}^{3}$.

The expression Eq. (32) for the multiplier, gives the following representations Eq. (12) of the non-trivial arrow fields $X_{f}, f \in \operatorname{Hom}(\mathcal{Q})$,

$$
\begin{aligned}
\hat{a}\left(f_{1}\right):\left(\psi_{A} ; \psi_{B_{1}}, \psi_{B_{2}}\right) & \mapsto\left(\psi_{B_{1}} ; \psi_{B_{1}}, \psi_{B_{2}}\right) \\
\hat{a}\left(f_{2}\right):\left(\psi_{A} ; \psi_{B_{1}}, \psi_{B_{2}}\right) & \mapsto\left(\psi_{B_{2}} ; \psi_{B_{1}}, \psi_{B_{2}}\right) \\
\hat{a}(g):\left(\psi_{A} ; \psi_{B_{1}}, \psi_{B_{2}}\right) & \mapsto\left(\psi_{A} ; \psi_{A}, \psi_{A}\right) \\
\hat{a}(r):\left(\psi_{A} ; \psi_{B_{1}}, \psi_{B_{2}}\right) & \mapsto\left(\psi_{A} ; \psi_{B_{1}}, \psi_{B_{1}}\right) \\
\hat{a}(s):\left(\psi_{A} ; \psi_{B_{1}}, \psi_{B_{2}}\right) & \mapsto\left(\psi_{A} ; \psi_{B_{2}}, \psi_{B_{2}}\right) .
\end{aligned}
$$

Note that arrows with the same domain and range are now separated, as required.

Of course, it is easy to write these operators as $3 \times 3$ matrices. For example,

$$
\hat{a}\left(f_{1}\right):\left(\begin{array}{l}
a \\
b \\
c
\end{array}\right) \mapsto\left(\begin{array}{l}
b \\
b \\
c
\end{array}\right)=\left(\begin{array}{lll}
0 & 1 & 0 \\
0 & 1 & 0 \\
0 & 0 & 1
\end{array}\right)\left(\begin{array}{l}
a \\
b \\
c
\end{array}\right)
$$

so that

$$
\hat{a}\left(f_{1}\right)=\left(\begin{array}{lll}
0 & 1 & 0 \\
0 & 1 & 0 \\
0 & 0 & 1
\end{array}\right)
$$

with the adjoint

$$
\hat{a}\left(f_{1}\right)^{\dagger}=\left(\begin{array}{ccc}
0 & 0 & 0 \\
1 & 1 & 0 \\
0 & 0 & 1
\end{array}\right)
$$

If we forget the causal structure on the set $B$, then there is the additional arrow $p: B \rightarrow B$ defined in Eq. (52). The corresponding arrow field $X_{p}$ is represented in the quantum theory by

$$
\hat{a}(p):\left(\psi_{A} ; \psi_{B_{1}}, \psi_{B_{2}}\right) \mapsto\left(\psi_{A} ; \psi_{B_{2}}, \psi_{B_{1}}\right)
$$

\section{Further Quantum Operations}

\subsection{Combining Causal Sets}

There are some special operations on causal sets (posets) that one might wish to incorporate in the present scheme. For example [3]: 
1. If $A$ and $B$ are disjoint posets, the disjoint union $A \cup B$ is defined on the set-theoretic disjoint union by the ordering $x \leq y$ if

(a) $x, y \in A$ and $x \leq_{A} y$; or

(b) $x, y \in B$ and $x \leq_{B} y$.

2. If $A$ and $B$ are disjoint posets, the linear sum $A \oplus B$ is defined on the set-theoretic disjoint union with the ordering $x \leq y$ if

(a) $x, y \in A$ and $x \leq_{A} y$; or

(b) $x, y \in B$ and $x \leq_{B} y$; or

(c) $x \in A$ and $y \in B$.

3. If $A$ and $B$ are posets, the set-theoretic Cartesian product $A \times B$ can be given a poset structure by defining $(x, y) \leq(p, q)$ if $x \leq_{A} p$ and $y \leq_{B} q$.

Of course, these constructions are only of use if the category concerned includes them. For example, the disjoint union of two connected posets is disconnected, and therefore would not be included if $\mathcal{Q}$ is a category of connected causal sets. On the other hand, the linear sum of connected posets is connected, and therefore could be used.

Let us start with the linear sum. In a simple quantisation scheme with complex-valued state functions on $\mathrm{Ob}(\mathcal{Q})$, it is natural to define operators $\hat{D}_{B}, B \in \mathrm{Ob}(\mathcal{Q})$, by

$$
\left(\hat{D}_{B} \psi\right)(A):=\psi(A \oplus B)
$$

for all $A \in \operatorname{Ob}(\mathcal{Q})$. These represent the $\oplus$ operation in the sense that $\hat{D}_{C} \hat{D}_{B}=\hat{D}_{C \oplus B}$.

Things are more complicated when multipliers are used since it is necessary to construct an appropriate linear map from $\mathcal{K}[A \oplus B]$ to $\mathcal{K}[A]$. To do this within the category quantisation scheme we need to pick some preferred arrow from $A$ to $A \oplus B$. The obvious choice is the injection $i_{A, A \oplus B}: A \rightarrow A \oplus B$, which leads to the definition

$$
\left(\hat{D}_{B} \psi\right)(A):=\kappa\left(i_{A, A \oplus B}\right) \psi(A \oplus B) .
$$

Of course, the same thing can be done for the disjoint union: i.e., use the natural embedding of $A$ in $A \cup B$.

For the product operation, the analogy of Eq. (70) is

$$
\left(\hat{\Pi}_{B} \psi\right)(A):=\psi(A \times B) .
$$


However, the analogue of Eq. (71) needs more thought. This is because the natural map associated with a product $A \times B$ is the projection $\pi_{A \times B, A}$ : $A \times B \rightarrow A,(a, b) \mapsto a$. But the linear map $\kappa\left(\pi_{A \times B, A}\right)$ is then from $\mathcal{K}[A]$ to $\mathcal{K}[A \times B]$, which is in the wrong direction. The obvious resolution is to use the adjoint $\kappa\left(\pi_{A \times B, A}\right)^{\dagger}$, which maps $\mathcal{K}[A \times B]$ to $\mathcal{K}[A]$. Thus we define

$$
\left(\hat{\Pi}_{B} \psi\right)(A):=\kappa\left(\pi_{A \times B, A}\right)^{\dagger} \psi(A \times B) .
$$

By these means, the causal-set operations of direct sum, disjoint union, and product can be incorporated into the category quantisation scheme.

Clearly, in a general category of small sets $\mathcal{Q}$, analogues of Eq. (71) and Eq. (73) can be used to represent the operations of taking, respectively, the disjoint union, and product, of two sets providing these exist as objects in $\mathcal{Q}$.

\subsection{When $\operatorname{Ob}(\mathcal{Q})$ has the Structure of a Lattice}

A mathematically similar situation to the poset operations in Section 4.1 arises in the context of an older approach to quantum topology discussed in [4]. This involved quantising the set of topologies $\tau(X)$ on a fixed set $X$ by exploiting the fact that $\tau(X)$ is an algebraic lattice. ${ }^{7}$ In general, if the set of objects $\operatorname{Ob}(\mathcal{Q})$ of a small category $\mathcal{Q}$ is a lattice, then the ' $V$ ' and ' $\wedge$ ' lattice operations can be incorporated into the category quantisation scheme in direct analogy with equations Eq. (71) and Eq. (73). Thus, for the $\vee$ operation we exploit the canonical arrow $o_{A, A \vee B}: A \rightarrow A \vee B$ (where $o_{x, y}: x \rightarrow y$ is the arrow associated with $x \leq y$ ) and define

$$
\left(\hat{O}_{B} \psi\right)(A):=\kappa\left(o_{A, A \vee B}\right) \psi(A \vee B),
$$

while, for the $\wedge$ operation, we use the arrow $o_{A \wedge B, A}: A \wedge B \rightarrow A$ and define

$$
\left(\hat{\Pi}_{B} \psi\right)(A):=\kappa\left(o_{A \wedge B, A}\right)^{\dagger} \psi(A \wedge B) .
$$

It is easy to check that, by these means, the lattice operations are represented in the quantum theory in the sense that

$$
\hat{O}_{B} \hat{O}_{C}=\hat{O}_{B \vee C}
$$

\footnotetext{
${ }^{7}$ Within the context of our present discussion, this earlier scheme is deficient in the sense that multipliers were not used, and hence the internal structure of each topology $\tau \in \tau(X)$ is not represented. It would be interesting to revisit that earlier work in the light of the more sophisticated category quantisation techniques.
} 
and

$$
\hat{\Pi}_{B} \hat{\Pi}_{C}=\hat{\Pi}_{B \wedge C}
$$

A special case is the lattice of subsets of some master set $U$. The quantisation scheme discussed in Section 3 can then be augmented with operations

$$
\begin{aligned}
\left(\hat{O}_{B} \psi\right)(A) & :=\kappa\left(o_{A, A \cup B}\right) \psi(A \cup B) \\
\left(\hat{\Pi}_{B} \psi\right)(A) & :=\kappa\left(o_{A \cap B, A}\right)^{\dagger} \psi(A \cap B)
\end{aligned}
$$

for all $A, B \subset U$, which are the analogues of Eq. (74) and Eq. (75) respectively. In a similar way, one can implement the operation of taking the complement of a set in which $A \mapsto U-A$.

\section{Quantum Field Theory in this Language}

Standard quantum field theory can also be discussed in the language of quantising on a category, and provides another example (albeit, somewhat contrived) of how the formalism works.

We start by considering what is superficially the same category as in Eq. (20), but with the object $n$ now being identified with the vector space $\mathbb{R}^{3 n}$. This gives the chain

$$
\{0\} \stackrel{i_{0}}{\rightarrow} \mathbb{R}^{3} \stackrel{i_{1}}{\rightarrow} \mathbb{R}^{6} \rightarrow \cdots \rightarrow \mathbb{R}^{3(n-1)} \rightarrow \cdots
$$

where $i_{k}: \mathbb{R}^{3 k} \rightarrow \mathbb{R}^{3(k+1)}$ is the natural embedding of vector spaces

$$
\begin{aligned}
\mathbb{R}^{3 k} & \stackrel{i_{k}}{\rightarrow} \mathbb{R}^{3(k+1)} \\
\left(x_{1}, x_{2}, \ldots, x_{3 k}\right) & \mapsto\left(x_{1}, x_{2}, \ldots, x_{3 k}, 0,0,0\right)
\end{aligned}
$$

If the corresponding quantum theory is viewed as being of a system whose configuration space can be any these vector spaces, then each $\mathbb{R}^{3 k}$ has the Euclidean group $E(3 k)$ as its group of isometries. As such, it is a natural choice for the monoid of arrows from the object $\mathbb{R}^{3 k}$ to itself. Thus once again we have a situation in which the arrows in the original category can be extended by including appropriate transformations of the internal structures of the objects. Thus, in this example it is reasonable to define the most general arrow from $\mathbb{R}^{3 k}$ to $\mathbb{R}^{3(k+1)}$ to be of the form $\beta \circ i_{k} \circ \alpha$ where $\alpha \in E(3 k)$ and $\beta \in E(3(k+1))$.

The monoids (actually, groups) $\operatorname{Hom}\left(\mathbb{R}^{3 k}, \mathbb{R}^{3 k}\right) \simeq E(3 k)$ can represented in the quantum theory by associating to each vector space $\mathbb{R}^{3 k}, k \geq 1$, the 
Hilbert space $L^{2}\left(\mathbb{R}^{3 k}\right)$, and associating to the null vector space $\{0\}$ the space $\mathbb{C}$. This Hilbert bundle can be turned into a contravariant presheaf by defining the linear operators $\kappa\left(i_{k}\right): L^{2}\left(\mathbb{R}^{3(k+1)}\right) \rightarrow L^{2}\left(\mathbb{R}^{3 k}\right)$ as

$$
\left(\kappa\left(i_{k}\right) \phi\right)\left(x_{1}, x_{2}, \ldots, x_{k}\right):=\phi\left(x_{1}, x_{2}, \ldots, x_{k}, 0,0,0\right)
$$

for all $\phi \in L^{2}\left(\mathbb{R}^{3(k+1)}\right)$. More generally, the arrow $\beta \circ i_{k} \circ \alpha: \mathbb{R}^{3 k} \rightarrow \mathbb{R}^{3(k+1)}$ is associated with the multiplier $R^{3 k}(\alpha) \kappa\left(i_{k}\right) R^{3(k+1)}(\beta): L^{2}\left(\mathbb{R}^{3(k+1)}\right) \rightarrow$ $L^{2}\left(\mathbb{R}^{3 k}\right)$ where $R^{3(k+1)}(\beta)$ denotes the representation of $\beta \in E(3(k+1))$ on $L^{2}\left(\mathbb{R}^{3(k+1)}\right)$, and $R^{3 k}(\alpha)$ denotes the representation of $\alpha \in E(3 k)$ on $L^{2}\left(\mathbb{R}^{3 k}\right)$.

A cross-section of this Hilbert bundle corresponds to an element of bosonic Fock space, defined as $\mathbb{C} \oplus L^{2}\left(\mathbb{R}^{3}\right) \oplus L^{2}\left(\mathbb{R}^{6}\right) \oplus \cdots$, and so we are close to recovering non-relativistic, bosonic quantum field theory. However, in this category approach there is no obvious way of deriving the usual annihilation and creation operators of quantum field theory. The problem is that this particular category does not have enough arrows between different objects.

This suggests to try again using the chain Eq. (20)

$$
0 \rightarrow 1 \rightarrow 2 \rightarrow \cdots \rightarrow n-1 \rightarrow \cdots
$$

but now define the arrows from an object $k$ to $l(l>k)$ to be functions in $L^{2}\left(\mathbb{R}^{3(l-k)}\right)$, i.e., $\operatorname{Hom}(k, l):=L^{2}\left(\mathbb{R}^{3(l-k)}\right)$. Composition of arrows/functions is then defined as the tensor product operation. More precisely, if $f: j \rightarrow k$ and $g: k \rightarrow l$, so that $f \in L^{2}\left(\mathbb{R}^{3(k-j)}\right)$ and $g \in$ $L^{2}\left(\mathbb{R}^{3(l-k)}\right)$, then $g \circ f: j \rightarrow l$ is defined as $g \circ f:=g \otimes f \in L^{2}\left(\mathbb{R}^{3(l-k)}\right) \otimes$ $L^{2}\left(\mathbb{R}^{3(k-j)}\right) \simeq L^{2}\left(\mathbb{R}^{3(l-j)}\right)$. This arrow composition law is associative since $f \otimes(g \otimes h)=(f \otimes g) \otimes h$ for functions $f, g, h$. When $k=l$, it is consistent to identify $L^{2}\left(\mathbb{R}^{3(l-k)}\right)$ with the vector space $\mathbb{C}$; i.e., for all $n \geq 0$, we set $\operatorname{Hom}(n, n):=\mathbb{C}$, with composition of complex numbers being defined as multiplication.

Multipliers are needed to separate the arrows between different objects, and the first step is to construct the bundle of Hilbert spaces over $\mathrm{Ob}(\mathcal{Q})=$ $\mathbb{N}$ in which the fibre $\mathcal{K}[n]$ over $n \in \mathrm{Ob}(\mathcal{Q})$ is $L^{2}\left(\mathbb{R}^{3 n}\right)$ for $n>0$, and with $\mathcal{K}[0] \simeq \mathbb{C}$. Then, if $f: k \rightarrow l$, so that $f \in L^{2}\left(\mathbb{R}^{3(l-k)}\right)$, we define $\delta(f): \mathcal{K}[k] \rightarrow \mathcal{K}[l]$ by $\delta(f)(h):=f \otimes h$, for all $h \in \mathcal{K}[k] \simeq L^{2}\left(\mathbb{R}^{3 k}\right)$. Note that $f \in L^{2}\left(\mathbb{R}^{3}\right)$ defines an arrow $f: k \rightarrow k+1$ for all $k>0$, and the associated (covariant) multiplier $\delta(f): L^{2}\left(\mathbb{R}^{3 k}\right) \rightarrow L^{2}\left(\mathbb{R}^{3(k+1)}\right)$ is the usual Fock space creation operator $\hat{b}(f)^{\dagger}$.

By these means we have constructed a covariant presheaf, rather than the contravariant one used earlier. A contravariant presheaf can be obtained 
by defining the multiplier $\kappa(f): \mathcal{K}[l] \rightarrow \mathcal{K}[k], f: k \rightarrow l$, as the adjoint of $\delta(f)$. This can then be used to define the operators $\hat{a}(X)$ as in Eq. (12).

Alternatively, the adjoint $\hat{a}(X)^{\dagger}$ can be defined directly using $\delta$. Specifically, as shown in I., the general expression for the adjoint operator $\hat{a}(X)^{\dagger}$ is

$$
\left(\hat{a}(X)^{\dagger} \psi\right)(B)=\sum_{A \in \ell_{X}^{-1}\{B\}} m(X, A)^{\dagger} \psi(A)
$$

for all $A \in \mathrm{Ob}(\mathcal{Q})$. In the present case, this becomes

$$
\begin{aligned}
\left(\hat{a}(X)^{\dagger} \psi\right)(n) & =\sum_{k \in \ell_{X}^{-1}\{n\}} \delta(X(k)) \psi(k) \\
& =\sum_{\left\{k \mid n=\ell_{X} k\right\}} X(k) \otimes \psi(k)
\end{aligned}
$$

In particular, if $f \in L^{2}\left(\mathbb{R}^{3}\right)$, and if we define the arrow field $X^{f}(n):=$ $f: n \rightarrow n+1$ for all $n \geq 0$, then

$$
\begin{aligned}
\left(\hat{a}\left(X^{f}\right)^{\dagger} \psi\right)(n) & =f \otimes \psi(n-1) \\
& =\hat{b}(f)^{\dagger} \psi(n-1)
\end{aligned}
$$

for all $n \geq 1$.

Note that although the objects have no internal structure, it is still of course true that the Hilbert space $L^{2}\left(\mathbb{R}^{3 n}\right)$ carries a representation of the Euclidean group $E(3 n)$ (and, indeed, of the canonical commutation relations) in $3 n$ dimensions.

\section{Conclusions}

We have seen how the general theory of quantising on a category $\mathcal{Q}$ can be applied to two different situations. The first is when the category is a poset, in which case the objects have no internal structure, and a quantisation with complex-valued state functions is sufficient.

The second, and more important, situation was one of the original motivations for studying the category quantisation scheme: namely, quantisation on a category whose objects are sets that could be potential models for space-time (or space). In particular, we have shown how to construct suitable multipliers for a category of finite sets. We have also indicated how this scheme might be extended to arbitrary sets equipped with measures. 
A major task for future research is to return to the examples of sets with structure - such as posets, or topological spaces - to get a better idea of when the irreducible representations of the category quantisation monoid of the underlying category of sets remain irreducible when the arrows are restricted to be structure-preserving maps. It is not clear how much can be said about this issue in general, and how much will depend on the details of the specific category. A useful step in this direction would be to study a variety of simple models of quantum casual sets and quantum topologies.

Another important topic for the future is the addition of matter degrees of freedom to a quantum causal set theory. In doing so, it will be necessary to amalgamate the insights into the local Hilbert spaces $\mathcal{K}[A], A \in \mathrm{Ob}(\mathcal{Q})$, obtained from the present paper and $\mathbf{I}$., with the understanding of the nature of quantum history theory as given by the 'history projection operator' (HPO) approach to consistent history theory. In the HPO approach, to any causal set $A$ there would be associated a certain Hilbert space $\mathcal{J}(A)$ whose projection operators represent propositions about the matter variables in the background space-time $A$. The task is to combine these Hilbert spaces $\mathcal{J}(A)$ with the Hilbert spaces $\mathcal{K}[A]$ of the quantum causal set theory in an appropriate way. The resulting quantum history theory will then involve propositions about the matter fields and the space-times in which they propagate.

Alternatively, one could start with a canonical approach to quantising on a causal background - for example, as discussed in [5] - and then use the techniques in the present paper to allow the background itself to be 'quantised'.

In either case, it must be emphasised that the techniques developed in the present paper constitute only a 'toolkit' for constructing such theories. The hard task is to use these tools to build physically realistic models of 'quantum space' or 'quantum space-time'.

\section{Acknowledgements}

Support by the EPSRC in form of grant GR/R36572 is gratefully acknowledged. 


\section{References}

[1] C. J. Isham, A new approach to quantising space-time: I. Quantising on a general category. Adv. Theor. Phys. 7 331-367 (2003). gr-qc/0303060.

[2] C. J. Isham, A new approach to quantising systems: III. State vectors as functions on arrows. (2003).

[3] B. A. Davey and H. A. Priestley, Introduction to Lattices and Order. Cambridge University Press, Cambridge, (1990).

[4] C. J. Isham, Quantum topology and quantization on the lattice of topologies. Class. Qu. Grav. 6, 1509-1534, (1989).

[5] L. Hawkins., F. Markopoulou and H. Sahlmann Evolution in quantum causal histories. hep-th/0302111, (2003). 